\title{
Douleurs neuropathiques et santé mentale
}

\section{Neuropathic Pain and Mental Health}

Peut-on dépister précocement la douleur neuropathique ? Quels sont les traitements mis à disposition des patients ? Quelles sont les avancées médicales dans le domaine ? Le Pr Nadine Attal, spécialiste en neurologie et algologie, répond à ces questions dans ce septième numéro de Douleur et Santé mentale édité par l'Institut UPSA de la douleur.

Les douleurs neuropathiques sont l'ensemble des douleurs résultant d'une lésion ou d'une maladie du système somatosensoriel.

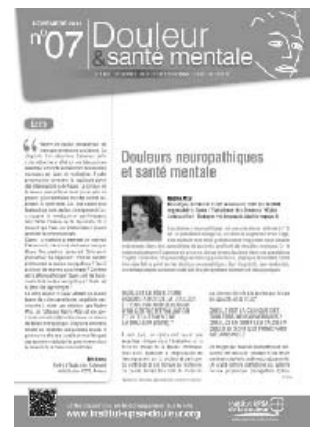

En France, la population atteinte de douleurs neuropathiques est estimée à $7 \%$. Cependant, ces douleurs sont peu connues chez les patients souffrant de troubles mentaux. La méconnaissance et l'absence de prise en charge de ces douleurs peuvent engendrer à long terme une aggravation physique et mentale du patient.

\section{Des symptômes au diagnostic}

On différencie la douleur chronique de la douleur neuropathique grâce à des symptômes bien distincts. Les douleurs neuropathiques sont le plus souvent associées à des douleurs continues telles que les brûlures ou des douleurs paroxystiques. Des douleurs provoquées par des simulations mécaniques légères, comme le frottement des draps, ou encore des douleurs thermiques peuvent également apparaître.

Du fait de la particularité de ces symptômes, la douleur neuropathique est considérée à présent comme une entité clinique à part entière.

Le diagnostic est exclusivement clinique. Ces dernières années, des outils de dépistage des douleurs neuropathiques, tels que le questionnaire DN4, ont été développés.

\section{Traitement médicamenteux et non médicamenteux}

La prise en charge d'un patient souffrant de douleurs neuropathiques est délicate. En effet, la douleur ne répond pas aux antalgiques classiques, le traitement repose alors sur quelques antidépresseurs et antiépileptiques entraînant parfois certains effets indésirables.

La simulation électrique transcutanée et la stimulation magnétique transcrânienne répétitive sont utilisées dans certains centres pour répondre aux douleurs neuropathiques. De plus, des techniques complémentaires comme l'hypnose ou l'acupuncture peuvent être proposées.

\section{Les avancées dans le domaine du traitement de la douleur neuropathique}

Des études épidémiologiques en population générale ont été réalisées pour mieux évaluer la réponse thérapeutique. L'avenir est prometteur grâce au développement de nombreuses molécules de traitements telles que les antagonistes du $\mathrm{NGF}^{1}$ ou de l'angiotensine 2 .

\section{Comment se procurer cette publication?}

Cette publication, comme l'ensemble de la documentation proposée par l'institut, est disponible gracieusement en libre téléchargement sur le site Internet de l'Institut UPSA de la douleur : www.institut-upsadouleur.org.

\footnotetext{
$\overline{{ }^{1} \text { Antagonistes }}$ du facteur de croissance neuronale ou nerve growth factor.
} 